\title{
Endoscopic Diagnosis of Colorectal Neoplasms Using Autofluorescence Imaging
}

Yoji Takeuchi, M.D., Noriya Uedo, M.D., Masao Hanafusa, M.D., Noboru Hanaoka, M.D., Sachiko Yamamoto, M.D., Ryu Ishihara, M.D., Hiroyasu Iishi, M.D.

Department of Gastrointestinal Oncology, Osaka Medical Center for Cancer and Cardiovascular Diseases, Osaka, Japan

\begin{abstract}
Many techniques have been developed to reduce the number of missed lesions during colonoscopy screening. Autofluorescence imaging (AFI) is one of the newly developed image-enhanced endoscopy (IEE) techniques, which functions similar to narrow band imaging (NBI) and flexible spectral imaging color enhancement (FICE), that can improve the detection and characterization of both polypoid and non-polypoid colonic neoplasms by enhancing their macroscopic features. We have previously reported that AFI, when used in combination with a transparent hood mounted on the tip of the endoscope to maintain distance from the colonic mucosa, results in the detection of approximately 1.6 times more colorectal neoplasms than conventional white light (WLI) colonoscopy. We have also revealed that AFI results in a higher flat neoplasm detection rate than WLI. Because the images of colorectal lesions visualized using AFI differ between histological lesion types, AFI also offers the possibility of differentiating neoplastic from non-neoplastic lesions. However, the difference between neoplastic and non-neoplastic lesions in the images generated using AFI relies on the density of the magenta coloring of the image and is therefore somewhat subjective. Recent studies suggest that NBI with magnification may be a superior modality for characterizing the neoplastic status of small colonic polyps. Although further developments are needed, the recent development of IEEs allows us to efficiently detect and differentiate colorectal neoplasms during colonoscopy screening. This article reviews the use of AFI in the diagnosis of colorectal neoplasms and discusses its advantages and limitations. (Intest Res 2012;10:142-151)
\end{abstract}

Key Words: Colonoscopy; Colonic Neoplasms

\section{INTRODUCTION}

Colorectal cancer is a leading cause of cancer deaths worldwide. ${ }^{1,2}$ Both the incidence and mortality due to colorectal cancer have been increasing rapidly in Korea. According to the Korean Central Cancer Registry, colorectal cancer is the third most common cancer in Korea. ${ }^{3}$ Transition through the adenoma-carcinoma sequence is thought to be the major route of development of colorectal cancer $^{4}$ and mortality due to colorectal cancer can be reduced by removal of colorectal adenomas.,

Received November 7, 2011. Revised November 14, 2011. Accepted November 16, 2011.

- Correspondence to: Yoji Takeuchi, M.D., Department of Gastrointestinal Oncology, Osaka Medical Center for Cancer and Cardiovascular Diseases, 1-3-3 Nakamichi, Higashinari-ku Osaka 537-8511, Japan

Tel: +81-6-6972-1181, Fax: +81-6-6981-4067

E-mail: takeuti-yo@mc.pref.osaka.jp
Therefore, early diagnosis and removal of colorectal neoplasms (both adenomas and early cancerous lesions) are of obvious importance. Colonoscopy is one of the most reliable methods for both diagnosis and removal of colorectal neoplasms. ${ }^{7}$ However, in a study that investigated adenoma diagnosis miss rates using back-toback colonoscopy, Rex et al. ${ }^{8}$ reported that colonoscopists missed approximately $24 \%$ of adenomas during conventional colonoscopy screening. In addition, the importance of non-polypoid type, slightly elevated or depressed colorectal neoplasms in the development of colorectal cancer is increasingly being recognized. These slightly elevated or depressed lesions, which develop from a 'de novo' pathway ${ }^{9,10}$ rather than the adenomacarcinoma sequence, are difficult to detect using conventional white light imaging (WLI) colonoscopy. ${ }^{11}$ Therefore, it is necessary to develop a more sensitive method for the diagnosis of colorectal neoplasms, espe- 
cially for non-polypoid, slightly elevated and depressed lesions.

Chromoendoscopy, which is a conventional WLI colonoscopy with dye spraying, is currently considered as an essential procedure for the detection and characterization of gastrointestinal neoplasms in Japan. However, as it is more technically troublesome and time consuming than WLI, it is not routinely used worldwide. The development of an equally effective and more technically facile diagnostic method of endoscopy has been anticipated. Autofluorescence imaging (AFI) is one of several newly developed image-enhanced endoscopy (IEE) techniques. ${ }^{12,13}$ These techniques, which also include narrow band imaging (NBI) ${ }^{14,15}$ and flexible spectral imaging color enhancement, ${ }^{16,17}$ enhance the macroscopic features of gastrointestinal neoplasms making endoscopists easier to detect and differentiate. ${ }^{18}$ AFI is a promising technique for improving the sensitivity of diagnosis of non-polypoid colorectal neoplasms. There are many reports on the efficacy of AFI for upper gastrointestinal endoscopy, ${ }^{18-23}$ and there have been several studies investigating the diagnosis of colorectal neoplasms using AFI. In this article, we will review the available literature on the use of AFI for the diagnosis of colorectal neoplasms.

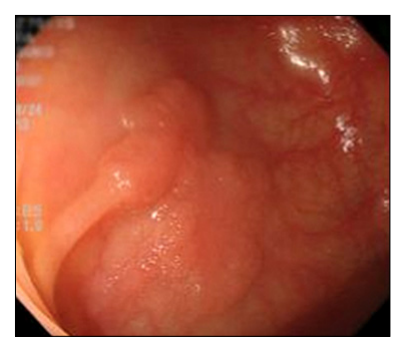

White light imaging

\section{Excitation light}

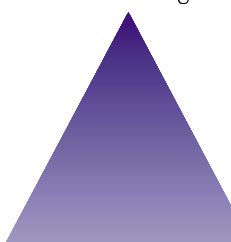

Autofluorescence

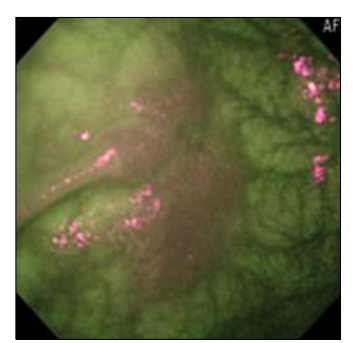

Autofluorescence imaging

- Mucosal thickness

- Hb concentration

- Inflammation, etc.
Colon

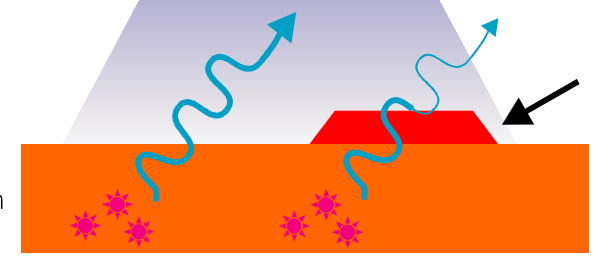

Fluorophores (collagen, NADH, etc.)
Fluorescence is the emission of a longer wavelength of light from a substance called a fluorophore following irradiation of that fluorophore by a short wavelength excitation light. In the gastrointestinal tract, endogenous fluorophores such as collagen, nicotinamide adenine dinucleotide, flavin, and porphyrins exist in both the mucosa and submucosa. When a short wavelength excitation light illuminates the gastrointestinal mucosa, natural tissue fluorescence called autofluorescence is emitted from these endogenous fluorophores. ${ }^{24}$ The AFI video-endoscopy system produces real-time pseudocolor images with emitted autofluorescence. Since the AFI video-endoscopy system utilizes only natural tissue fluorescence from endogenous fluorophores, it does not require any drug administration or dye spraying and thus reduces the risk of adverse side effects.

Detection of abnormal lesions using AFI videoendoscopy relies on differences in the autofluorescence intensity or spectrum between normal and abnormal gastrointestinal mucosa. These differences in the autofluorescence intensity or spectrum are affected by the concentration or depth distribution of endogenous fluorophores and by differences in tissue micro-architecture, including altered mucosal thickness or blood (hemoglobin) concentration. Autofluorescence is generally re- 
duced by protruding lesions when compared with the normal mucosa (Fig. 1). ${ }^{25}$ These differences in fluorescence, which are mainly different in intensity, appear as color differences in the AFI video-endoscopy images. ${ }^{26}$

\section{HOW TO USE AFI VIDEO-ENDOSCOPY}

Early AFI system was a fiberoptic endoscope with a heavy image-intensifying camera unit attached to the endoscope tip. However, these provide poor image quality and poor maneuverability and thus not suitable for general clinical usage in the era of video-endoscopy. The latest AFI system uses a dedicated video-endoscope (CF-FQ260AZI; Olympus Medical Systems, Tokyo, Japan) that incorporates two charge-coupled devices (CCDs) for the AFI and WLI modes. Its appearance and maneuverability are almost identical to those of a conventional video-colonoscope. Each mode can rapidly be selected by pushing a button on the control head of colonoscope. The AFI system is the first imaging system that detects autofluorescence by a CCD incorporated within the video-endoscope.

In the AFI mode, a blue excitation light for inducing autofluorescence $(395-475 \mathrm{~nm})$, and a green (G'-) light $(550 \mathrm{~nm})$ for generating reflected images, are produced sequentially from the light source via a rotary filter. An excitation light cut filter is incorporated with the CCD of the AFI mode; this permits only 490 to $625 \mathrm{~nm}$ wavelength light to filter through, allowing tissue autofluorescence and reflected $G$ '-light to reach the CCD while blocking blue excitation light. When the G'-light is illuminated, reflected images are visualized. The image processor artificially colors the autofluorescence images to green and the green reflected image to red and blue, and composite images are displayed on the video screen (Fig. 2). The intensity of the reflected G'-light is determined by the absorption features of hemoglobin. Normal mucosa emits bright autofluorescence, and thus the composite color appears bright green. Protruding lesions (e.g., tumors, polyps) absorb autofluorescence and appear as magenta, which is the complementary color of green. As hemoglobin absorbs both autofluorescence and $\mathrm{G}^{\prime}$-light $(550 \mathrm{~nm})$ areas containing hemoglobin are displayed as dark green in the AFI mode.

In the WLI mode, the light source provides red, green, and blue wavelength light sequentially using a rotary filter. As the CCD for the WLI mode is the same as that in the conventional high-definition colonoscope the EVIS CF-H260AZI (Olympus, Tokyo, Japan), diagnosis of colorectal neoplasm can be done using both the AFI mode and the high-definition WLI mode.

\section{HOW TO WATCH COLORECTAL NEOPLASMS USING AFI}

When using AFI, normal colorectal mucosa without

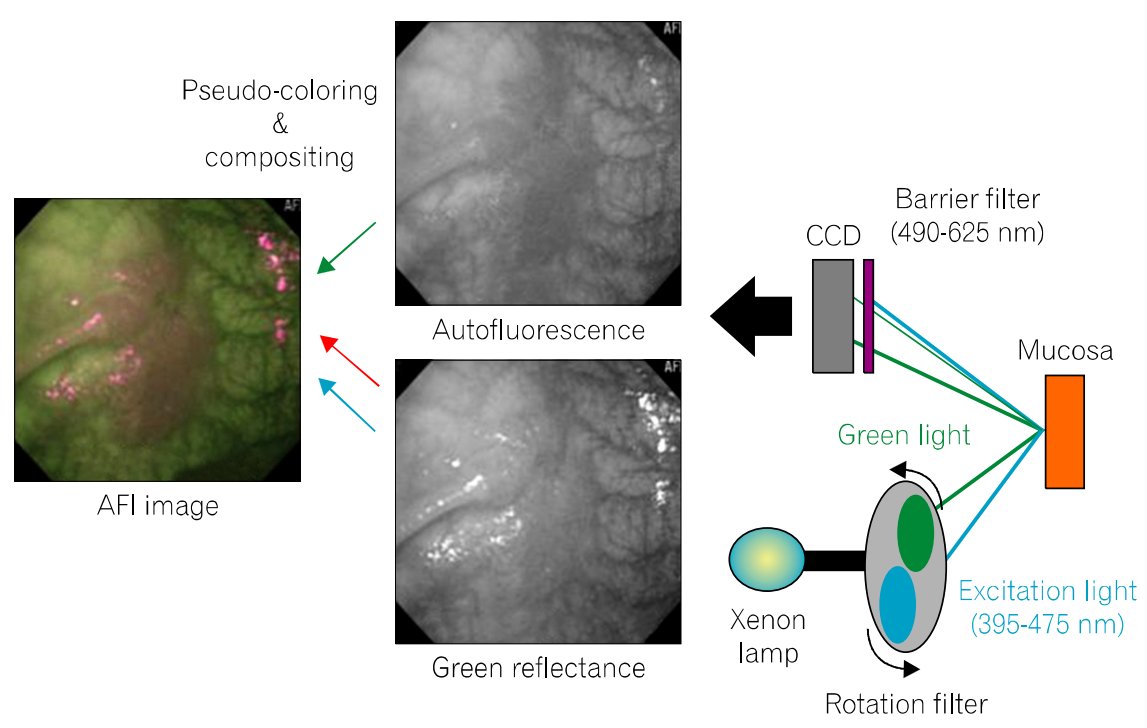

Fig. 2. Diagram of the autofluorescence imaging (AFI) system. CCD, charge-coupled device. 
inflammation appears green in color. Images of colorectal lesions differ according to macroscopic lesion type $^{27}$ and it is important to understand the difference in appearance of the various lesion types when using the AFI colonoscope. The macroscopic types of colorectal neoplasms are variable and they can be divided into three categories; polypoid, flat, and depressed types. ${ }^{28}$

Polypoid lesions (Paris classification Type 0-I), which are the most common macroscopic type of colorectal neoplasms, are easily detectable with conventional WLI. They appear as polypoid magenta lesions when using AFI (Fig. 3).

Slightly elevated lesions (Paris classification Type 0 -IIa) are an uncommon macroscopic type of colorectal neoplasm. Slightly elevated lesions are usually similar in color or reddish compared to the surrounding mucosa and can be difficult to recognize using conventional WLI. If these are overlooked they can be a cause of interval cancer (invasive colorectal cancer which occurs after a negative screening colonoscopy). When visualized using AFI, slightly elevated lesions appear as a distinct magenta area within the surrounding green mucosa (Fig. 4).

Depressed lesions (Paris classification Type 0-IIc) are rare macroscopic findings of colorectal cancer. ${ }^{29,30} \mathrm{De}$ pressed lesions are thought to arise through a de novo pathogenic sequence and demonstrate early invasive characteristics. It is difficult to identify depressed lesions using conventional WLI colonoscopy. We have reported a successful AFI imaging of a diminutive, depressed-type early colon cancer invading the sub-
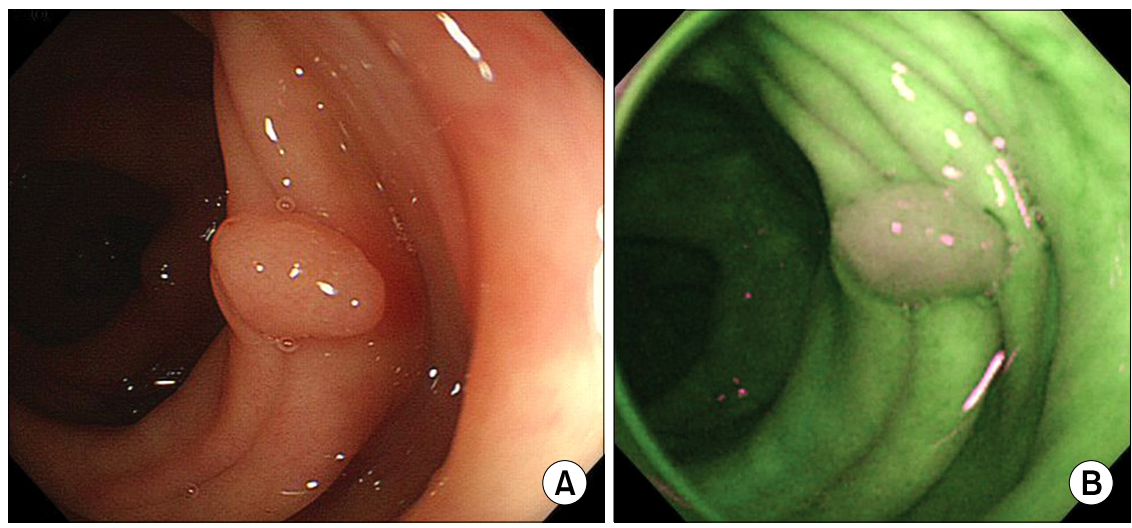

Fig. 3. Endoscopic images of a polypoid adenoma. (A) White light image of a polypoid adenoma in the ascending colon. (B) The autofluorescence image of a polypoid adenoma in the ascending colon. The tumor appears as a polypoid magenta lesion.
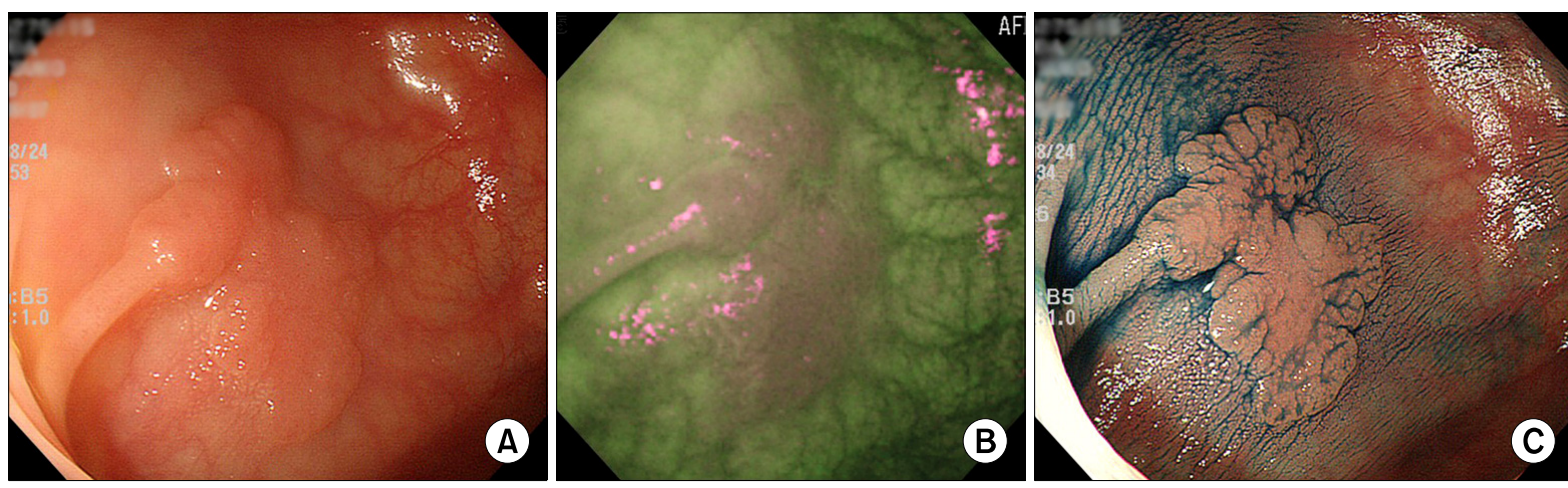

Fig. 4. Endoscopic images of a slightly elevated adenoma. (A) White light image of a slightly elevated adenoma in the transverse colon. The lesion is similar in color to the surrounding mucosa and cannot be seen clearly. (B) The autofluorescence imaging (AFI) image of a slightly elevated adenoma in the transverse colon. The lesion appears as a distinct magenta area within the surrounding green mucosa and can be seen clearly. (C) Chromoendoscopic image of a flat adenoma in the transverse colon using $0.2 \%$ indigo carmine. The macroscopic features of the adenoma are enhanced by the indigo carmine which resembles the AFI image. 

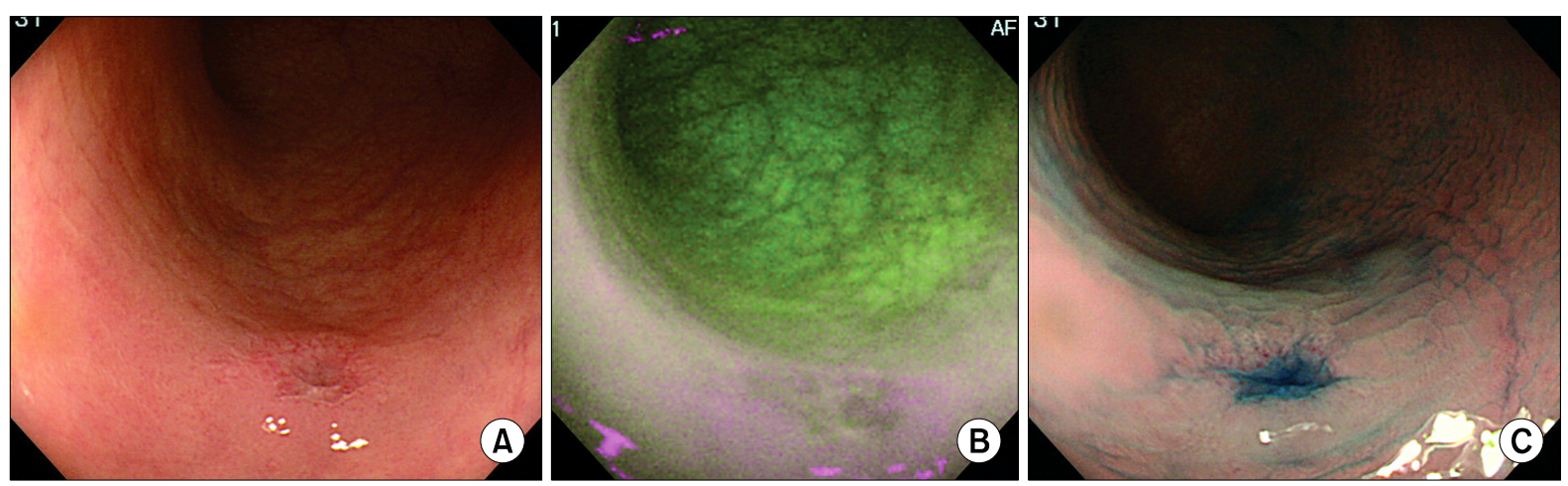

Fig. 5. Endoscopic images of a depressed-type early colon cancer. (A) White light image of a depressed lesion in the sigmoid colon. The lesion is similar in color to the surrounding mucosa. It is difficult to detect using conventional colonoscopy. (B) The autofluorescence imaging (AFI) image of a depressed lesion in the sigmoid colon. The lesion appears as a green area within surrounding magenta mucosa. The depressed lesion is thinner than the surrounding mucosa and the intensity of the autofluorescence is not reduced. Therefore, the depressed area appears green while the surrounding mucosa, which is relatively thicker than the depressed area, appears magenta. (C) Chromoendoscopic image of a depressed lesion in the sigmoid colon using $0.2 \%$ indigo carmine. This image resembles the corresponding AFI image. The lesion had invaded into the submucosal layer.

mucosal layer. The lesion appeared as a $5 \mathrm{~mm}$ green area surrounded by magenta mucosa (Fig. 5), which differs from the appearance of other types of colorectal neoplasms visualized using AFI. ${ }^{31}$ Depressed lesions are thinner than the surrounding mucosa and the intensity of autofluorescence is not reduced. Therefore, the depressed area appears green while the surrounding mucosa, which is relatively thicker than the depressed area, appears magenta.

\section{ADENOMA DETECTION RATE OF AFI}

AFI enhances visualization of colorectal neoplasms, which appear with a distinct color difference relative to normal colorectal mucosa. Inoue et al. ${ }^{32}$ visualized 49 lesions in 43 patients using WLI, AFI, and chromoendoscopy with $0.2 \%$ indigo carmine visualization of the surface appearance and circumferential margin of the lesions which revealed better with AFI than with WLI. Therefore, it is expected that the use of AFI will improve the detection rate of colorectal lesions during colonoscopy screening, especially in detecting slightly elevated lesions that are difficult to detect using WLI.

In a pilot study that evaluated the feasibility of AFI for the detection of colorectal adenomas in 64 patients, we compared the adenoma detection rates of AFI and
Table 1. Comparison of Two Different Pilot Studies for the Detection of Colorectal Lesions Using WLI and AFI

\begin{tabular}{|c|c|c|c|c|}
\hline \multirow[t]{2}{*}{ Author } & \multicolumn{2}{|c|}{ Matsuda et al. ${ }^{34}$} & \multicolumn{2}{|c|}{ Uedo et al. $^{33}$} \\
\hline & WLI & AFI & WLI & $\mathrm{AFI}$ \\
\hline Patients & \multicolumn{2}{|c|}{167} & \multicolumn{2}{|c|}{64} \\
\hline Investigated area & \multicolumn{2}{|c|}{$\begin{array}{l}\text { Right sided } \\
\text { colon }\end{array}$} & \multicolumn{2}{|c|}{$\begin{array}{l}\text { Left sided } \\
\text { colon }\end{array}$} \\
\hline Study design & \multicolumn{2}{|c|}{$\begin{array}{c}\text { Modified back- } \\
\text { to-back RCT }\end{array}$} & \multicolumn{2}{|c|}{$\begin{array}{c}\text { Modified back- } \\
\text { to-back RCT }\end{array}$} \\
\hline Detected lesion & 73 & 100 & 57 & 58 \\
\hline Detected neoplasm & 69 & 92 & 28 & 26 \\
\hline Missed lesion & 45 & 19 & 13 & 12 \\
\hline Missed protruded lesion & 6 & 5 & 7 & 10 \\
\hline Missed flat lesion & 39 & 14 & 6 & 2 \\
\hline
\end{tabular}

WLI, white light imaging; AFI, autofluorescence imaging; $\mathrm{RCT}$, randomized controlled trial.

Values are number.

WLI using back-to-back sigmoidoscopy. Each method detected almost the same number of polyps and adenomas, and their sensitivity and specificity were almost identical (Table 1). Therefore, we concluded that AFI did not demonstrate superior efficacy to WLI for detecting lesions in the sigmoid colon. ${ }^{33}$

Contrary to these results, Matsuda et al. ${ }^{34}$ reported that AFI detected polyps in the right-sided colon better than 
WLI. They compared the adenoma miss rate using AFI and WLI in 167 patients who underwent a modified back-to-back colonoscopy of the right-sided colon including the cecum, ascending and transverse colon. The miss rate for all polyps with AFI (30\%) was significantly less than that with WLI $(49 \%)(P=0.01$, Table 1$)$.

The differences between the results of these two studies may be due to the investigated area of the colon in each study (Table 1). The image quality of AFI, i.e., resolution, refresh rate and speed of brightness control, is worse than that of WLI, and it is necessary to observe the colonic mucosa slowly and carefully to recognize the color differences between lesions and the surrounding mucosa. However, it is often difficult to keep the colonoscope moving slowly in the narrow and tortuous sigmoid colon. This may have impeded our ability to fully utilize the potential of AFI in the sigmoid colon. However, the right-sided colon is wider and relatively straighter in comparison to the sigmoid colon, and thus may provide more suitable conditions for AFI. Furthermore, slightly elevated lesions had a tendency to be missed by WLI and be detected by AFI in both pilot studies (Table 1). We concluded that AFI has superior efficacy to WLI for detecting slightly elevated lesions, but its utility is still limited in certain areas of the colon.

\section{AFI AS A COLONOSCOPY SCREENING METHOD}

Rex et $a .^{8}$ reported that the number of adenomas missed during colonoscopy was greater in the right-sided $(27 \%)$ than the left-sided $(21 \%)$ colon, but the difference was not significant. Therefore, we thought that the superior ability of AFI to detect adenomas when compared with WLI should be shown in the left as well as the right side of the colon. Attachment of a transparent hood (TH) to the tip of an AFI colonoscope can help to maintain an adequate distance between the tip and the mucosa and thus allow the CCD to catch reflected autofluorescence, even in the narrow and tortuous sigmoid colon. Therefore, we attached a TH to the tip of an AFI colonoscope during colonoscopy screening using AFI. ${ }^{35}$

We then conducted a randomized controlled trial to evaluate the efficacy of AFI with a $\mathrm{TH}^{36}$ In this trial, we used a two-by-two factorial design to investigate the impact of AFI and the impact of a TH on neoplasm detection rates (Fig. 6). Patients undergoing colonoscopy screening for detailed investigation of positive fecal occult blood test or surveillance after endoscopic removal of colorectal adenomas were enrolled and randomly assigned to one of four groups (WLI alone, WLI+TH, AFI alone, AFI+TH, Fig. 7). Patients were excluded if (1) they had a history of colectomy or major abdominal

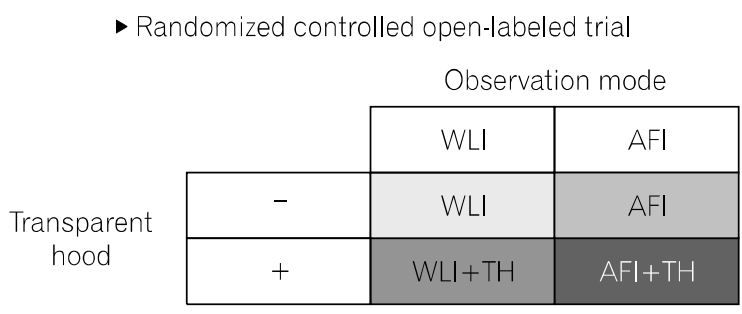

Fig. 6. The concept of a $2 \times 2$ factorial design used to simultaneously investigate the efficacy of the autofluorescence imaging (AFI) and of mounting a transparent hood $(\mathrm{TH})$ on the endoscope tip. WLI, white light imaging.

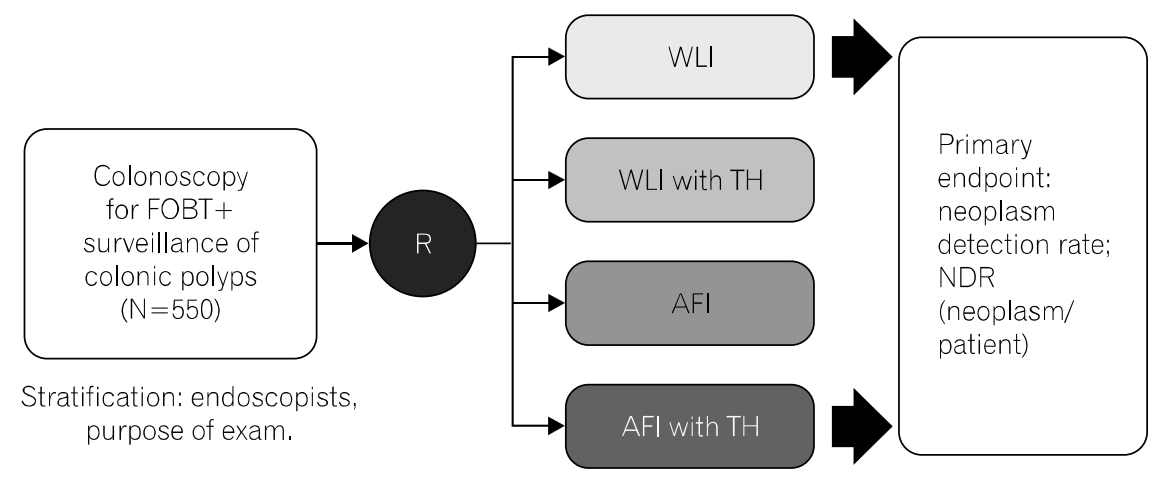

Fig. 7. Design of a randomized, controlled, $2 \times 2$ factorial trial for investigation of the impact of the autofluorescence imaging (AFI) and use of a transparent hood (TH) on neoplasm detection rates. FOBT, fecal occult blood test; NDR, neoplasm detection rate (detected neoplasms per patient); WLI, white light imaging, $\mathrm{R}$, randomization. 


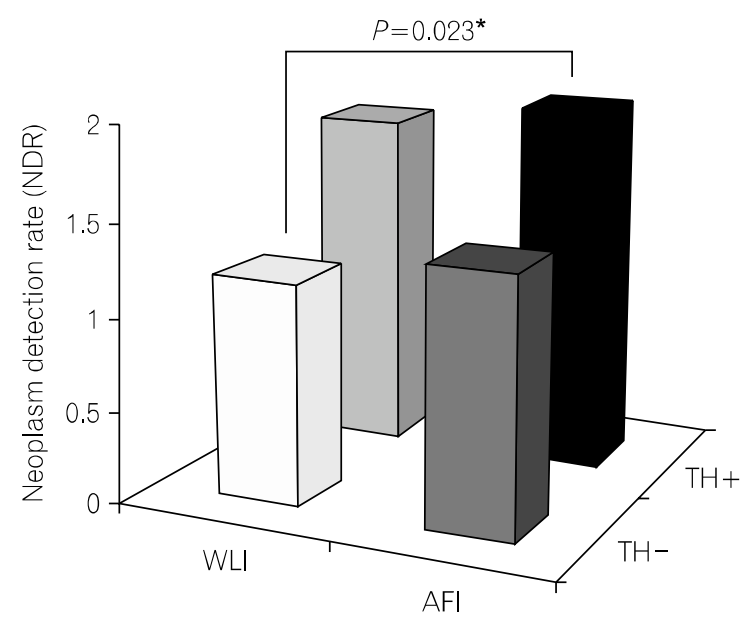

surgery; (2) they had symptoms suggestive of colorectal stenosis or cancer; (3) they had inflammatory bowel disease, familial polyposis, or known colorectal cancer; (4) they had severe organ failure, non-correctable coagulopathy, or were undergoing anticoagulant therapy; or (5) the colonoscopist judged that they did not understand the meaning of random allocation. The primary endpoints of this study were neoplasm detection rate (number of detected neoplasms perpatient) by WLI without a TH (control group) and by AFI with a TH.

Between November 2008 and November 2009, 923 patients were scheduled to undergo colonoscopy in our endoscopy unit. A total of 362 patients were excluded from enrollment as per the exclusion criteria listed above, leaving a total of 561 patients who were assigned randomly to the different groups. A total of 1,105 lesions were detected in 380 patients. Specimens were not obtained from 13 lesions, and histological diagnosis was available for 1,092 lesions. Eight hundred and seventy-five lesions were diagnosed as neoplasms and 217 were diagnosed as non-neoplastic. There were 383 (69\%) patients with detected lesions and 329 (59\%) of patients had neoplasms.

The primary endpoint, neoplasm detection rate (number of detected neoplasms per patient $[95 \% \mathrm{CI}]$ ) in the $\mathrm{AFI}+\mathrm{TH}$ group, was significantly higher than in the group that underwent WLI alone (1.96 [1.50-2.43] vs 1.19 [0.93-1.44], respectively $P=0.023$ [Tukey-Kramer multiple comparison method]). AFI+TH detected more neoplasms than conventional colonoscopy (Fig. 8). In

\begin{tabular}{|c|c|}
\hline Allocation & $\begin{array}{c}\text { NDR } \\
{[95 \% . C . I]}\end{array}$ \\
\hline $\mathrm{WLI}$ & $\begin{array}{c}1.19 \\
{[0.93-1.44]}\end{array}$ \\
\hline $\mathrm{WLI}+\mathrm{TH}$ & $\begin{array}{c}1.72 \\
{[1.28-2.15]}\end{array}$ \\
\hline $\mathrm{AFI}$ & $\begin{array}{c}1.36 \\
{[1.07-1.65]}\end{array}$ \\
\hline $\mathrm{AFI}+\mathrm{TH}$ & $\begin{array}{c}1.96 \\
{[1.50-2.43]}\end{array}$ \\
\hline
\end{tabular}

Fig 8. The primary endpoint of the randomized, controlled trial. Neoplastic lesion detection rate (NDR) in the autofluorescence imaging (AFI) with a transparent hood (AFI+ transparent hood $[\mathrm{TH}])$ group was significantly higher than in the white light imaging (WLI) alone group. *Tukey-kramer multiple comparison method.

subgroup analysis using a Poisson regression model, polypoid neoplasm detection rate was higher when a $\mathrm{TH}$ was used (independent of AFI or WLI) than when a $\mathrm{TH}$ was not used. In addition, the use of AFI resulted in a higher flat neoplasm detection rate than WLI. Based on these results, we concluded that the combination of AFI with a TH is efficacious for the detection of colorectal neoplasms, and that a TH should be attached to the colonoscope tip when using AFI for colonoscopy screening.

\section{PROBLEMS TO BE SOLVED}

We have reported that the combination of AFI with a $\mathrm{TH}$ results in the detection of approximately 1.6 times more colorectal neoplasms than conventional WLI colonoscopy. However, the median size of the lesions detected by AFI with a TH was only $4.1 \mathrm{~mm}$, and almost all of them (98\%) were indolent non-neoplastic lesions or low-grade adenomas. Although early detection and resection of colorectal adenomas is an efficacious and fundamental strategy for prevention of colorectal cancer development, use of such a sensitive modality for detection of colorectal neoplasms can increase the cost, time and labor required for formal histopathological diagnosis of detected indolent neoplasms. Thus, highly sensitive colonoscopy techniques may result in a higher rate of colorectal neoplasm histopathological diagnosis that does not translate into a comparatively higher rate of cancer prevention and cure. 
In 2009, Ignjatovic et al. ${ }^{37}$ proposed the 'DISCARD' policy in Lancet Oncology. The principle of the policy is that 'optical diagnosis' distinguishing neoplastic from non-neoplastic lesions using NBI without magnification, can be a real-time virtual alternative to formal histopathological diagnosis of small polyps. This policy can lead to substantial savings in the cost, time and labor required for formal histopathological diagnosis by discarding small colorectal polyps with 'optical diagnosis' using NBI. We believe that this proposal has a merit as in vivo 'optical diagnosis' of small colorectal lesions which lead to potential clinical and cost advantages.

AFI also offers the possibility of 'optical diagnosis' since the images of colorectal lesions generated using AFI differ according to the macroscopic, as well as the histological types. Hyperplastic lesions appear as light magenta in color, which differs from the appearance of neoplastic lesions as shown in Fig. 9. Arita et al. ${ }^{38}$ re- ported that the color-contrast index of lesions visualized using AFI increased as the malignant potential increased (with hyperplastic polyps showing less color-contrast than adenomas, which in turn show less color-contrast than carcinomas), and they concluded that quantitative analysis of autofluorescence intensity using the color-contrast index is helpful in discriminating different types of colorectal mucosal lesions (Table 2). Sato et al. ${ }^{39}$ also reported that AFI can discriminate colon adenomas from hyperplastic polyps. In a prospective multicenter study, they found that the accuracy of AFI for distinguishing adenomas from hyperplastic lesions was $84.9 \%$ (Table 2).

Although AFI is a useful tool for 'optical diagnosis', which discriminates of neoplastic and non-neoplastic colorectal lesions, Kuiper et al. ${ }^{40}$ reported that the sensitivity, specificity and accuracy of AFI in differentiating adenomas from non-adenomatous lesions were $90 \%$,
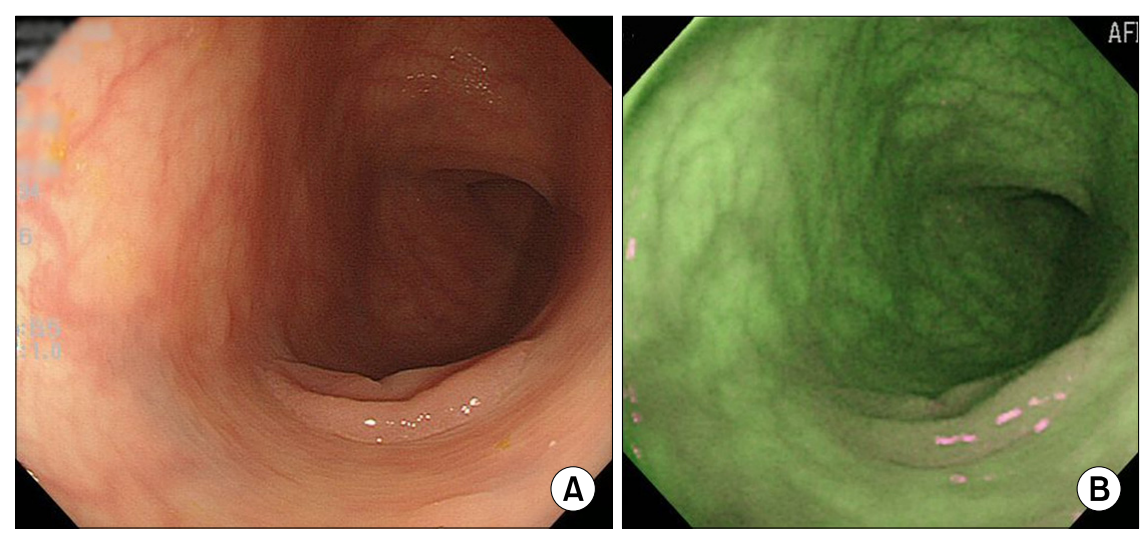

Fig. 9. Endoscopic images of a hyperplastic polyp. (A) White light image of a slightly elevated hyperplastic polyp in the sigmoid colon. (B) The autofluorescence imaging image of a slightly elevated hyperplastic polyp in the sigmoid colon. The lesion appears as a light magenta area within the surrounding green mucosa. The color difference between the lesion and the surrounding mucosa is less than the color difference between a neoplastic lesion and the surrounding mucosa.

Table 2. Diagnostic Performance for Distinguishing Neoplastic Lesion from Non-neoplastic Lesion

\begin{tabular}{|c|c|c|c|c|c|c|c|c|c|c|}
\hline \multirow{2}{*}{$\begin{array}{c}\text { Author } \\
\text { Mode }\end{array}$} & \multirow{2}{*}{ 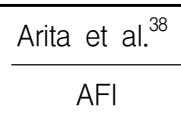 } & \multicolumn{3}{|c|}{ Sato et al. $^{39}$} & \multicolumn{2}{|c|}{ Kuiper et al. ${ }^{40}$} & \multicolumn{4}{|c|}{ Ignjatovic et al. ${ }^{41}$} \\
\hline & & HRE & AFI & $\mathrm{NBI}$ & AFI & $\mathrm{NBI}$ & WLE & AFI & $\mathrm{NBI}$ & $\mathrm{NBI}-\mathrm{ME}$ \\
\hline Lesion (n) & 54 & & 424 & & 239 & 238 & \multicolumn{4}{|c|}{80} \\
\hline Sensitivity & 0.95 & 0.84 & 0.89 & 0.91 & 0.90 & 0.87 & 0.69 & 0.63 & 0.74 & 0.93 \\
\hline Specificity & 0.64 & 0.47 & 0.67 & 0.77 & 0.37 & 0.63 & 0.60 & 0.43 & 0.56 & 0.59 \\
\hline
\end{tabular}

AFI, autofluorescence imaging; HRE, high resolution endoscopy; NBI, narrow-band imaging; WLE, white light endoscopy; NBI-ME, narrow-band imaging with magnification. 
$37 \%$, and $62 \%$, respectively. The accuracy of AFI was deemed insufficient for clinical use (Table 2). Furthermore, Ignjatovic et al. ${ }^{41}$ reported that AFI did not show better sensitivity, specificity, or accuracy for characterizing small colonic polyps compared to those of WLI in their trial. Since the difference between non-neoplastic and neoplastic lesions in the images generated using AFI relies on the density of the magenta coloring of the image, distinguishing between lesion types can be subjective. In the same study by Ignjatovic et al., ${ }^{41}$ NBI with magnification (NBI-ME) had the best overall accuracy and interobserver agreement when compared to WLI, AFI and NBI without magnification (Table 2). Thus NBI-ME might be more suitable than AFI for 'optical diagnosis'. Furthermore, characterization of the capillary pattern on the surface of detected lesions using NBI-ME has been reported to allow assessment of the degree of dysplasia in early colorectal neoplasia. ${ }^{42}$ By incorporating this technique so called NBI-ME for the 'detect inspect characterise resect and discard (DISCARD)' policy, it is possible to distinguish high-grade adenomas from low-grade adenomas or non-neoplastic lesions. This allows us to adopt a more accurate and detailed 'DISCARD' policy-the 'DISCARD-ME' policy. We have already completed enrolling participants in the phase II study to investigate the efficacy of the 'DISCARD-ME' policy.

\section{CONCLUSION}

Although AFI has the ability to detect small lesions missed with WLI, at this time the utility of AFI for 'optical diagnosis' is limited. This may be due to the low resolution of current AFI video-endoscopy systems, or to the subjective nature of interpretating color density of the images. Further improvement of AFI image quality, or additional color-enhancing technologies related to AFI, are needed. Otherwise, other imageenhancing endoscopy techniques (e.g., NBI-ME) might be superior in performing an 'optical biopsy' in practice.

\section{REFERENCES}

1. Jemal A, Bray F, Center MM, Ferlay J, Ward E, Forman D. Global cancer statistics. CA Cancer J Clin 2011;61:212-236.
2. Center MM, Jemal A, Ward E. International trends in colorectal cancer incidence rates. Cancer Epidemiol Biomarkers Prev 2009;18:1688-1694.

3. Annual Report of cancer incidence (2008) in Korea [Internet]. Goyang: National Cancer Center; [updated 2010; cited 2012]. Available from: http://ncc.re.kr/.

4. Morson B. President's address. The polyp-cancer sequence in the large bowel. Proc R Soc Med 1974;67:451-457.

5. Muto T, Bussey HJ, Morson BC. The evolution of cancer of the colon and rectum. Cancer 1975;36:2251-2270.

6. Winawer SJ, Zauber AG, Ho MN, et al. Prevention of colorectal cancer by colonoscopic polypectomy. The National Polyp Study Workgroup. N Engl J Med 1993;329:1977-1981.

7. Centers for Disease Control and Prevention (CDC). Vital signs: Colorectal cancer screening, incidence, and mortality--United States, 2002-2010. MMWR Morb Mortal Wkly Rep 2011; 60:884-889.

8. Rex DK, Cutler CS, Lemmel GT, et al. Colonoscopic miss rates of adenomas determined by back-to-back colonoscopies. Gastroenterology 1997;112:24-28.

9. Muto T, Nagawa H, Watanabe T, Masaki T, Sawada T. Colorectal carcinogenesis: historical review. Dis Colon Rectum 1997;40(10 Suppl):S80-S85.

10. Soetikno RM, Kaltenbach T, Rouse RV, et al. Prevalence of nonpolypoid (flat and depressed) colorectal neoplasms in asymptomatic and symptomatic adults. JAMA 2008;299:1027-1035.

11. Kahi CJ, Hewett DG, Rex DK. Relationship of non-polypoid colorectal neoplasms to quality of colonoscopy. Gastrointest Endosc Clin N Am 2010;20:407-415.

12. Kaltenbach T, Sano Y, Friedland S, Soetikno R; American Gastroenterological Association. American Gastroenterological Association (AGA) Institute technology assessment on imageenhanced endoscopy. Gastroenterology 2008;134:327-340.

13. Tajiri $\mathrm{H}$, Niwa $\mathrm{H}$. A new classification and precise definitions of endoscopic imaging. Gastroenterol Endosc 2009;51:1677-1685.

14. Gono K, Obi T, Yamaguchi M, et al. Appearance of enhanced tissue features in narrow-band endoscopic imaging. J Biomed Opt 2004;9:568-577.

15. Machida H, Sano Y, Hamamoto Y, et al. Narrow-band imaging in the diagnosis of colorectal mucosal lesions: a pilot study. Endoscopy 2004;36:1094-1098.

16. Miyake $\mathrm{Y}$, Nakaguchi T, Tsumura N, Yamataka $\mathrm{S}$, et al. Development of new electronic endoscopes using the spectral imaging of an internal organ. Proceedings of Thirteenth Color Imaging Conference; 2005 Nov; Scottsdale (AZ). Scottsdale: Society for Imaging Science and Technology; 2005. p. 261-263.

17. Mouri R, Yoshida S, Tanaka S, Oka S, Yoshihara M, Chayama $\mathrm{K}$. Evaluation and validation of computed virtual chromoendoscopy in early gastric cancer. Gastrointest Endosc 2009; 69:1052-1058.

18. Uedo N, Iishi H, Tatsuta $\mathrm{M}$, et al. A novel videoendoscopy system by using autofluorescence and reflectance imaging for diagnosis of esophagogastric cancers. Gastrointest Endosc 2005; 62:521-528.

19. Uedo N, Iishi H, Ishihara R, Higashino K, Takeuchi Y. Novel autofluorescence videoendoscopy imaging system for diagnosis of cancers in the digestive tract. Dig Endosc 2006;18(Suppl 1): S131-S136. 
20. Kato M, Uedo N, Ishihara R, et al. Analysis of the color patterns of early gastric cancer using an autofluorescence imaging video endoscopy system. Gastric Cancer 2009;12:219-224.

21. Inoue $\mathrm{T}$, Uedo $\mathrm{N}$, Ishihara $\mathrm{R}$, et al. Autofluorescence imaging videoendoscopy in the diagnosis of chronic atrophic fundal gastritis. J Gastroenterol 2010;45:45-51.

22. Hanaoka N, Uedo N, Shiotani A, et al. Autofluorescence imaging for predicting development of metachronous gastric cancer after Helicobacter pylori eradication. J Gastroenterol Hepatol 2010;25:1844-1849.

23. Ishihara R, Inoue $\mathrm{T}$, Hanaoka $\mathrm{N}$, et al. Autofluorescence imaging endoscopy for screening of esophageal squamous mucosal high-grade neoplasia: a phase II study. J Gastroenterol Hepatol 2012;27:86-90

24. Haringsma J, Tytgat GN, Yano H, et al. Autofluorescence endoscopy: feasibility of detection of GI neoplasms unapparent to white light endoscopy with an evolving technology. Gastrointest Endosc 2001;53:642-650.

25. Mayinger B, Jordan M, Horbach T, et al. Evaluation of in vivo endoscopic autofluorescence spectroscopy in gastric cancer. Gastrointest Endosc 2004;59:191-198.

26. Uedo $\mathrm{N}$, Ishihara $\mathrm{R}$, Iishi $\mathrm{H}$. Autofluorescence imaging videoendoscopy system for diagnosis of superficial gastric neoplasia. In: Niwa H, Tajiri H, Nakajima N, Yasuda K, eds. New challenges in gastrointestinal endoscopy. Japan: Springer, 2008. p. 191-199.

27. Takeuchi Y, Hanaoka N, Hanafusa M, et al. Autofluorescence imaging of early colorectal cancer. J Biophotonics 2011;4:490-497.

28. The Paris endoscopic classification of superficial neoplastic lesions: esophagus, stomach, and colon: November 30 to December 1, 2002. Gastrointest Endosc 2003;58(6 Suppl):S3-S43.

29. Kudo S, Tamure S, Nakajima T, et al. Depressed type of colorectal cancer. Endoscopy 1995;27:54-57.

30. Kaku E, Oda Y, Murakami Y, et al. Proportion of flat- and depressed-type and laterally spreading tumor among advanced colorectal neoplasia. Clin Gastroenterol Hepatol 2011;9:503-508.

31. Takeuchi Y, Uedo N, Higashino K, et al. Autofluorescence imaging of a diminutive, depressed-type early colon cancer invaded to the submucosal layer. Gastrointest Endosc 2010; 71:399-400.

32. Inoue $\mathrm{K}$, Wakabayashi $\mathrm{N}$, Morimoto $\mathrm{Y}$, et al. Evaluation of autofluorescence colonoscopy for diagnosis of superficial colorectal neoplastic lesions. Int J Colorectal Dis 2010;25: 811-816.

33. Uedo N, Higashino K, Ishihara R, Takeuchi Y, Iishi H. Diagnosis of colonic adenomas by new autofluorescence imaging system: a pilot study. Dig Endosc 2007;19(Suppl 1):S134-S138.

34. Matsuda T, Saito Y, Fu KI, et al. Does autofluorescence imaging videoendoscopy system improve the colonoscopic polyp detection rate?--a pilot study. Am J Gastroenterol 2008;103:1926-1932.

35. Takeuchi Y, Inoue T, Hanaoka N, Chatani R, Uedo N. Surveillance colonoscopy using a transparent hood and imageenhanced endoscopy. Dig Endosc 2010;22(Suppl 1):S47-S53.

36. Takeuchi $\mathrm{Y}$, Inoue $\mathrm{T}$, Hanaoka $\mathrm{N}$, et al. Autofluorescence imaging with a transparent hood for detection of colorectal neoplasms: a prospective, randomized trial. Gastrointest Endosc 2010;72:1006-1013.

37. Ignjatovic A, East JE, Suzuki N, Vance M, Guenther T, Saunders BP. Optical diagnosis of small colorectal polyps at routine colonoscopy (Detect InSpect ChAracterise Resect and Discard; DISCARD trial): a prospective cohort study. Lancet Oncol 2009;10:1171-1178.

38. Arita K, Mitsuyama K, Kawano H, et al. Quantitative analysis of colorectal mucosal lesions by autofluorescence endoscopy: discrimination of carcinomas from other lesions. Oncol Rep 2011;26:43-48.

39. Sato R, Fujiya M, Watari J, et al. The diagnostic accuracy of high-resolution endoscopy, autofluorescence imaging and narrow-band imaging for differentially diagnosing colon adenoma. Endoscopy 2011;43:862-868.

40. Kuiper T, van den Broek FJ, Naber AH, et al. Endoscopic trimodal imaging detects colonic neoplasia as well as standard video endoscopy. Gastroenterology 2011;140:1887-1894.

41. Ignjatovic A, East JE, Guenther $\mathrm{T}$, et al. What is the most reliable imaging modality for small colonic polyp characterization? Study of white-light, autofluorescence, and narrowband imaging. Endoscopy 2011;43:94-99.

42. Katagiri A, Fu KI, Sano Y, et al. Narrow band imaging with magnifying colonoscopy as diagnostic tool for predicting histology of early colorectal neoplasia. Aliment Pharmacol Ther 2008;27:1269-1274. 